\title{
Evolution of Pore Characteristics for Bentonite Modified by an Ionic Soil Stabilizer during Hydration Processes
}

\author{
Wei Huang $\mathbb{D}^{1}{ }^{1}$ Zili Feng, ${ }^{1}$ Huanran Fu, ${ }^{1}$ and Wei Xiang ${ }^{2}$ \\ ${ }^{1}$ Faculty of Airport Engineering and Transportation Management, Civil Aviation Flight University of China, \\ Guanghan 618307, China \\ ${ }^{2}$ Faculty of Engineering, China University of Geosciences, Wuhan 430074, China \\ Correspondence should be addressed to Wei Huang; huangwei@cafuc.edu.cn
}

Received 12 May 2021; Revised 12 August 2021; Accepted 9 September 2021; Published 4 October 2021

Academic Editor: Ming Hua

Copyright (c) 2021 Wei Huang et al. This is an open access article distributed under the Creative Commons Attribution License, which permits unrestricted use, distribution, and reproduction in any medium, provided the original work is properly cited.

An ionic soil stabilizer (ISS) is used to reinforce clay soils because the ISS can regulate the hydration processes and microstructures of clays. To evaluate the regulation of ISS, natural bentonite was modified by ISS at different concentrations in this research. Water vapour adsorption and X-ray diffraction (XRD) were carried out to interpret the hydration mechanism of bentonite. Meanwhile, an associated analysis between hydration pore structures and hydration mechanisms was implemented through variation of pore characteristic tests at different relative humidities (RHs) to distinguish multiscale pore adsorption of water during the corresponding hydration process. In addition, the pore characteristics were studied via XRD, nitrogen adsorption, and mercury injection tests. Finally, the origins that adsorbed water and pore structures changed by adding ISS were discussed. The results showed that for calcium bentonite, the cations hydrated first in the range of $0<\mathrm{RH}<0.45 \sim 0.55$, accompanied by the expansion of micropores. Then, adsorption occurred on the basal surface of the crystal layer in the range of $0.45 \sim 0.55<\mathrm{RH}$ $<0.8 \sim 0.9$, with water mainly adsorbed into the mesopores. With further hydration when $\mathrm{RH}>0.8 \sim 0.9$, diffused double layer (DDL) water ceaselessly entered the macropores. Both adsorbed water and multiscale pore size decreased when ISS was added to bentonite. The origins of the reduction were the regulation of ISS to exchangeable cations and the basal surface of the crystal layer.

\section{Introduction}

Swelling clay, which is intensely hydrophilic, has a highlighted behaviour in which clay expands with hydration and shrinks with dehydration. Generally, crystalline swelling and osmotic swelling occur sequentially when swelling clay hydrates [1-4]. At the beginning of hydration, water molecules are adsorbed into the clay interlayer, which is driven by the hydration energy of interlayer cations, resulting in the expansion of interlayer spacing $\left(d_{001}\right)$, which is no more than the thickness of the three water layers. This swelling discussion is commonly referred to as crystalline swelling [4]. With further adsorption of water, hydrated cations enter the liquid phase to generate a diffused double layer (DDL) around clay particles. Based on the repulsion of DDL, clay particles act over much longer distances. This swelling among particles is generally described as osmotic swelling
[3]. Thus, with clay swelling, the internal structure of the particles (interlayer space) and interparticle structure vary continuously.

According to the mechanism of adsorbing water, the two expansion stages actually originate from the unceasingly changing occurrence state of adsorbed water $[5,6]$. When adsorbing water, the internal and external surfaces of crystal layers and even the surface of clay particles generate water layers with varying hydration energy. Then, adsorbed water transforms from bound water to DDL water as the thickness of the water layer increases. In this hydration process, water molecules ceaselessly enter the interlayer and interparticle space, generating a "wedging force" to enter the space so that clay swells.

In return, the pores changing with clay swelling influence the processing of adsorbing water and the properties of adsorbed water, indicating that hydration and pores are 
reciprocal [7-9]. According to the nomenclature terms of the International Union of Pure and Applied Chemistry (IUPAC), the pores can be divided into micropores $(<2 \mathrm{~nm})$, mesopores $(2-50 \mathrm{~nm})$, and macropores $(>50 \mathrm{~nm})$ through their pore size. For bentonite, which comprises phyllosilicate minerals, micropores are distributed mainly in the interlamellar space $[5,6,10]$ and can be measured by X-ray diffraction (XRD) [11-13]. The mesopores are recognized in the space among particles and even in some space among aggregates $[5,6]$. This kind of pore is usually measured by vapour adsorption (e.g., water and nitrogen) based on multifarious pore analysis methods, such as the frequently used BJH [14] and DH [15] methods. Furthermore, macropores appear in the space among aggregates or even larger clay particles $[6,10]$, and the mercury intrusion method can be adopted to study this scale of pores [16].

As stated above, hydration and pore characteristics are interdependent and reciprocal. Therefore, a question about the associated evolution of "hydration pores" has arisen. Although the change in crystalline structure during hydration has been investigated by means of XRD and water adsorption $[12,13,17]$, the change in crystalline structure is concentrated on the microporous scale without mesoand macropores. In addition, a thermoporometry method has been employed to study the pore characterization of clay when it is hydrated [6]. This measurement could be made on liquid-saturated samples or on samples in equilibrium with a certain relative humidity ( $\mathrm{RH}$ or $\left.P / P_{0}\right)$ so that all the types of pores could be seen during clay hydration. However, the properties of adsorbed water in different pores are not defined clearly. Therefore, the associated evolutionary mechanism of "hydration pores" in the whole hydration process is discussed in this paper.

To solve the engineering problem that clay swelling brings while adsorbing water, many solutions have been proposed by students. Generally, cementitious materials such as lime and cement have been used to improve the engineering behaviours of clay $[18,19]$. These types of materials could interact with clay from the following two aspects: (1) bivalent $\mathrm{Ca}^{2+}$ in cementitious materials replaces the monovalent cations so that the hydrated film of clay soil is reduced; (2) the production of cementitious materials strengthens the connection among clay particles. However, $\mathrm{Ca}^{2+}$ could be replaced by other intense hydration cations in shifted water circumstances, resulting in damage to cementitious production. Additionally, the improvement has been proven to attenuate after several drying and wetting cycles [20]. To overcome these disadvantages, some new stabilizers have been developed, such as ionic soil stabilizers (ISSs), polymer stabilizers [21], and bioenzyme stabilizers [22]. In particular, ISS could regulate the hydration properties of clay particles, which has drawn extensive attention from students [23, 24]. With regard to the constituents, ISS is generally thought to be a kind of hydrosolvent that contains several dissociated ions. These ions could interact with clay minerals and change their surface properties. By regulating the hydration properties, ISS suppresses the expansive potential of swelling clay and improves its mechanical behaviours $[25,26]$.
The above results indicate that adding ISS to reinforce swelling clay was promising. However, most of these studies focused on comparative analysis of macroparameters for raw and modified samples, while the impacts that ISS had on the two key factors (hydration and pore) in the process of adsorbing water need to be investigated further. Therefore, in this paper, the associated evolutionary mechanism of hydration pores for bentonite in the whole hydration process is discussed first. Based on this discussion, the adsorbed water and pore characteristics of raw and modified bentonite were studied. Then, the modified mechanism of ISS could be revealed by understanding the variation in the hydration process and pore structure. These results could assist in the area of clay modification and improvement.

\section{Materials and Methods}

2.1. Clay Minerals and Sample Preparation. A natural bentonite from Greek volcanic areas was selected as the material to study in this paper. The phase composition by XRD (D8FOCUS with a $\mathrm{CuK}_{\alpha}$ radiation source, Ni filter, $40 \mathrm{kV}$, $40 \mathrm{~mA}$, scan range of $2-65^{\circ} 2 \theta$, step intervals of $0.02^{\circ} 2 \theta$, and dwell time of $2 \mathrm{~s}$; Bruker, Karlsruhe, Germany) demonstrated that this material extracted from physically crushed raw ore of bentonite and purified by hydraulic elutriation had above $95 \mathrm{wt} \%$ montmorillonite (Figure 1). GBT in the following discussion was short for this purified Greek bentonite. X-ray fluorescence (AXIOSmAX Instrument, PANalytical, Eindhoven, the Netherlands) tests showed that the chemical composition of this calcium bentonite dried at $105^{\circ} \mathrm{C}$ for 4 hours in mass percentage was $\mathrm{SiO}_{2}, 57.99 \%$; $\mathrm{Al}_{2} \mathrm{O}_{3}, 17.92 \% ; \mathrm{Fe}_{2} \mathrm{O}_{3}, 4.65 \% ; \mathrm{MgO}, 4.12 \%$; $\mathrm{CaO}, 3.86 \%$; $\mathrm{Na}_{2} \mathrm{O}, 0.59 \% ; \mathrm{K}_{2} \mathrm{O}, 0.45 \% ; \mathrm{MnO}, 0.07 \% ; \mathrm{TiO}_{2} 0.74 \% ; \mathrm{P}_{2} \mathrm{O}_{5}$, $0.19 \%$; and loss on ignition (LOI), 9.38\%. The cation exchange capacity (CEC) of GBT was determined by the barium chloride method, and the type and contents of exchangeable cations were measured through inductively coupled plasma-optical emission spectroscopy (iCAP 6300, Thermo Fisher Scientific, Waltham, Massachusetts, USA). The cation results (Table 1) showed that the main exchangeable cation was $\mathrm{Ca}^{2+}$, indicating that GBT was calcium bentonite. The Atterberg test showed that the liquid limit and plastic limit of this calcium GBT were $226.70 \%$ and $43.75 \%$, respectively.

2.2. Ionic Soil Stabilizer (ISS). The ionic soil stabilizer was developed independently by Pro. The Wei Xiang group was produced through sulfonation of cottonseed oil with concentrated sulfuric acid under certain chemical conditions. The main component of ISS was cottonseed oilbased sulfonated oil, which had few metal cations, such as $\mathrm{K}^{+}, \mathrm{Na}^{+}, \mathrm{Ca}^{2+}$, and $\mathrm{Mg}^{2+}$ (Table 2), demonstrating that ISS would not mislead the exchangeable cation analysis of modified bentonite.

2.3. Modified Samples by Adapting ISS. ISS was a kind of grease that could not interact with clay directly unless it was diluted by water at a certain volume ratio. In previous work on modifying natural expansive soil, we found that 


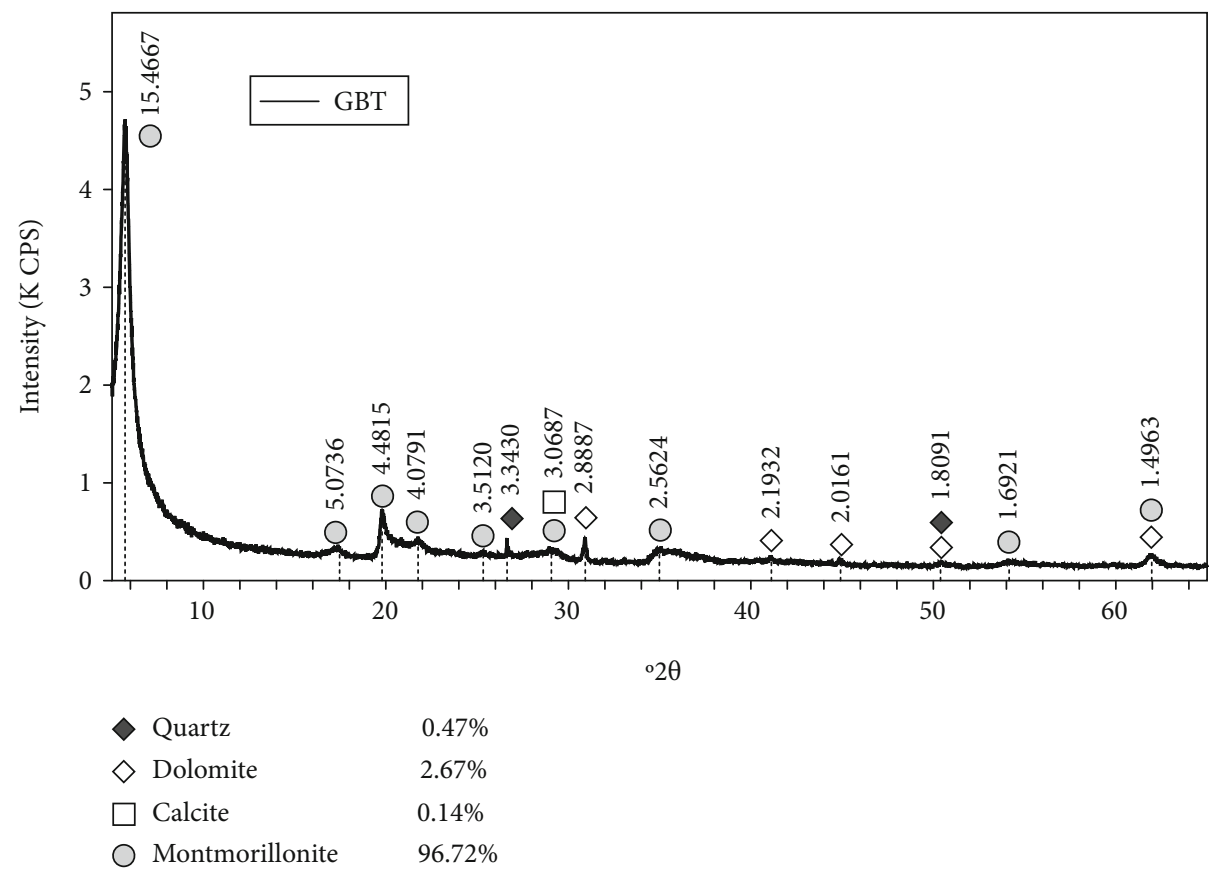

FIGURE 1: XRD spectrum of GBT.

TABLE 1: CEC and exchangeable cation content of GBT.

\begin{tabular}{lc}
\hline Contents $\left(\mathrm{meq} \cdot \mathrm{g}^{-1}\right)$ & $\mathrm{GMT}$ \\
\hline $\mathrm{CEC}$ & 0.7300 \\
$\mathrm{~K}^{+}$ & 0.0376 \\
$\mathrm{Na}^{+}$ & 0.1214 \\
$\mathrm{Ca}^{2+}$ & 0.4316 \\
$\mathrm{Mg}^{2+}$ & 0.1285 \\
Total bases & 0.7200 \\
\hline
\end{tabular}

TABLE 2: Metal cation content of ISS.

\begin{tabular}{lc}
\hline Contents $\left(\mathrm{meq} \cdot \mathrm{ml}^{-1}\right)$ & ISS \\
\hline $\mathrm{K}^{+}$ & 0.0008 \\
$\mathrm{Na}^{+}$ & 0.0047 \\
$\mathrm{Ca}^{2+}$ & 0.0099 \\
$\mathrm{Mg}^{2+}$ & 0.0026 \\
Total bases & 0.0181 \\
\hline
\end{tabular}

the optimal dilution ratio for ISS was $1: 200[27,28]$. In this paper, the material was purified bentonite with $>95 \mathrm{wt} \%$ clay minerals, which was 3.2 times the clay minerals of red clay $[27,28]$. Hence, higher dilution ratios (volume ratios of $1: 50$ and $1: 100$ ) were chosen to modify the bentonite. Then, $100 \mathrm{~g}$ of naturally air-dried GBT was selected to interact with $200 \mathrm{ml}$ of diluted ISS liquor. After sufficient stirring, the mixture of GBT and ISS was hermetically placed for 24 hours to acquire a complete interaction. Next, the modified bentonites were removed and leached to wash off the free ions and redundant ISS through centrifugation. This step was repeated 4 times to ensure that salt leaching was rigor- ous. Then, liquid nitrogen was added to pasty-modified bentonites to freeze-dry them. Finally, the freeze-dried samples were tested and designated GBT-1:50 and GBT-1:100 based on their ISS concentrations. Additionally, the raw bentonite tested was prepared in the same way and was designated GBT- 0 .

2.4. XRD and $d_{001}$ Reflections. Approximately $40 \mathrm{mg}$ GBT-0 was stirred in $0.7 \mathrm{ml}$ distilled water to become a suspension, which was spread on glass slides for air drying. To save experimental time, 14 identical slides for bentonites were made to be exposed to a certain $\mathrm{RH}$ at the same time. Then, all slides were dried in a muffle furnace at $350^{\circ} \mathrm{C}$ for $2 \mathrm{~h}$ to dehydrate all the adsorbed water in bentonite. After drying the bentonites, 14 slides were exposed to a certain $\mathrm{RH}$ (from 0 to 1 ) in airtight glass pots controlled by the desiccant and different saturated brine solutions for 30 days to ensure that the mass change of each slide was less than $0.0005 \mathrm{~g}$ and guarantee adsorption equilibrium. At the end, all the slides were stored in sealed cases separately and measured by the XRD method.

2.5. Water Vapour and Nitrogen Adsorption. The clastic freeze-drying materials (GBT-0, GBT-1:100, and GBT$1: 50)$ were put into an adsorption device named Autosorb-iQ (Quantachrome Instruments, Boynton Beach, Florida, USA) to measure water vapour adsorptiondesorption isotherms $\left(0<P / P_{0}<1\right)$ at $20^{\circ} \mathrm{C}$. Nitrogen adsorption-desorption isotherms $\left(0<P / P_{0}<1\right)$ were obtained through ASAP2460 (Micromeritics Instrument Corporation, Norcross, Georgia, USA). Before the adsorption test, each sample was vacuum dried at $105^{\circ} \mathrm{C}$ for 2 hours. 
2.6. Mercury Intrusion Method. The granular freeze-dried samples (GBT-0, GBT-1:100, and GBT-1:50) with a $5 \mathrm{~mm}$ grain size were placed in an AutoPore IV 9500 mercury injection apparatus (Micromeritics Instrument Corporation, Norcross, Georgia, USA) to investigate the macropores.

2.7. Variation of Pore Characteristic at Different RH. To obtain the variation in pore characteristics at different RHs, an indirect method was applied. First, three $20 \mathrm{~cm}^{3}$ compacted cutting ring samples of GBT- 0 were made with dry densities of $1.2 \mathrm{~g} / \mathrm{cm}^{3}, 1.3 \mathrm{~g} / \mathrm{cm}^{3}$, and $1.4 \mathrm{~g} / \mathrm{cm}^{3}$. After saturation, the compacted samples were placed in the airtight glass pot mentioned in Section 2.4 to desorb water. To save test time, other samples were put separately into another 13 airtight glass pots with different RHs. To guarantee desorption equilibrium, more than 3 months were taken, and the final mass change was less than $0.01 \mathrm{~g}$ in seven-day continuous weighing. Simultaneously, the volume of each compacted sample was measured and calculated by a Vernier caliper. Finally, the variation in void ratio at different RHs could be calculated through the specific gravity of bentonite particles using a value of $2.75 \mathrm{~g} / \mathrm{cm}^{3}$.

\section{Results and Discussions}

3.1. Hydration Mechanism of Bentonite. To better understand the influence of ISS on adsorbed water, the hydration process of natural bentonite was investigated first. Therefore, the hydration mechanism of raw bentonite is discussed as follows. The water vapour adsorption isotherm is the curve of adsorbance versus $\mathrm{RH}$ or $P / P_{0}$, which also describes the relationship between adsorbance and adsorption potential [29]. Therefore, this kind of isotherm implies information on the hydration energy of soil in a certain $\mathrm{RH}$ range. By comparing the shapes of the adsorption and desorption branches shown in Figure 2, they were obviously seen to be different. More specifically, the desorption branch appeared in a distinct stage with increasing $\mathrm{RH}$, while the adsorption branch changed more smoothly, demonstrating a clear transformation of hydration status and energy in the desorption process. Therefore, a desorption branch was selected to discuss the hydration mechanism.

Figure 3 shows the first derivative of adsorbed water content in the desorption branch versus $\mathrm{RH}$ ( or $P / P_{0}$ ), which was the water vapour adsorption velocity curve indicating a variation in the energy status of adsorbed water on bentonite [29]. This variation implied that inflection points appeared when the interaction between the soil and water changed, i.e., water molecules were adsorbed on clay with a new occupied form and energy status. There were obviously two peaks for GBT-0 in Figure 3, distributed in RH Sections $0.15 \sim 0.2$ and $0.8 \sim 0.9$, and the two peaks could be divided by $0.45 \sim 0.55$.

Water molecules were recognized to be able to interact with multifarious hydration sites (e.g., cations and the basal surface of the crystal layer) on the surface of clay, resulting in a difference in hydration energy for adsorbed water [30-32]. Moreover, the hydration energy of cations was much higher than the hydration energy of the basal surface, causing cation hydration to be the main driving force to adsorb water [11-13, 33, 34]. Thus, water molecules were supposed to move from the basal surface of the crystal layer with lower energy first during the desorption process. In addition, cations dehydrated afterwards. Hence, the first peak ranged from approximately 0.15 to 0.2 and the second peak ranged from approximately 0.8 to 0.9 in Figure 3, showing hydration of cations and adsorption of the basal surface, respectively. In addition, the RH Section $0.45 \sim 0.55$ was the boundary that distinguished cation and basal surface hydration.

To verify the hydration sequence above, the curve in which $d_{001}$ changes with RH $\left(P / P_{0}\right)$ for GBT-0 was studied through XRD (Figure 4). Additionally, some data cited from published references about calcium montmorillonite ( $\mathrm{Ca}$ Mont) are illustrated here [13, 35-37]. The results indicated that the curve form of GBT-0 was approximate to the curve of other calcium montmorillonites. Figure 4 shows that the interlayer spacing ( $d_{001}$ spacing) of GBT-0 and Ca-Monts increased with the samples adsorbing water and increased by approximately $2.7 \AA$, which was equal to the thickness of the monolayer of the water molecules when $\mathrm{RH}$ was close to 0.2 . With further hydration, the interlayer spacing increased to approximately one bilayer of water molecules when the RH was approximately 0.45 . Then, the $d_{001}$ spacing remained constant until the $\mathrm{RH}$ increased to 0.9 , indicating that the interlayers no longer swelled in the RH section.

The hydration energy of cations was higher than the hydration energy of the basal surface of the crystal layer (as mentioned above). Consequently, water molecules entered the interlayer and interacted with cations to form a hydration shell at the beginning of hydration, indicating that the driving force that caused water molecules to enter the interlayer was mainly from the hydration energy of cations. In the range of $0<\mathrm{RH}<0.15 \sim 0.2$, water molecules formed a monolayer of hydration shell of cations, and the $d_{001}$ spacing expanded by approximately $2.7 \AA$. Then, in the range of $0.15 \sim 0.2<\mathrm{RH}<0.45 \sim 0.55$, water molecules were adsorbed onto cations continuously to form the bilayer of the hydration shell of the cation to show the interlayer spacing of approximately $5.4 \AA$ sequentially. Furthermore, in the range of $0.45 \sim 0.55<\mathrm{RH}<0.8 \sim 0.9$, the $d_{001}$ spacing remained unchanged, implying that water molecules were adsorbed on the internal and external surfaces of the clay minerals. In this range, water molecules were adsorbed mainly on the basal surface or interspace among hydrated cations to form an integrated bilayer of water with the hydration shell of the cation; obviously, the interlayer spacing did not increase. Meanwhile, the interlamellar space could not expand infinitely with increasing $\mathrm{RH}$ because $\mathrm{Ca}^{2+}$ had a strong binding effect on lamellae so that the interlayer spacing remained unchanged until $\mathrm{RH} \approx 0.9$.

To summarize, the hydration mechanism for GBT- 0 and other Ca-Monts was as follows: (1) in the range of $0<\mathrm{RH}$ $<0.15 \sim 0.2$, interlamellar cations hydrated to form a monolayer of hydration shell of cations; (2) in the range of 0.15 $\sim 0.2<\mathrm{RH}<0.45 \sim 0.55$, interlayer cations hydrated sequentially to form a bilayer of hydration shell; (3) in the range of $0.45 \sim 0.55<\mathrm{RH}<0.8 \sim 0.9$, water molecules were 


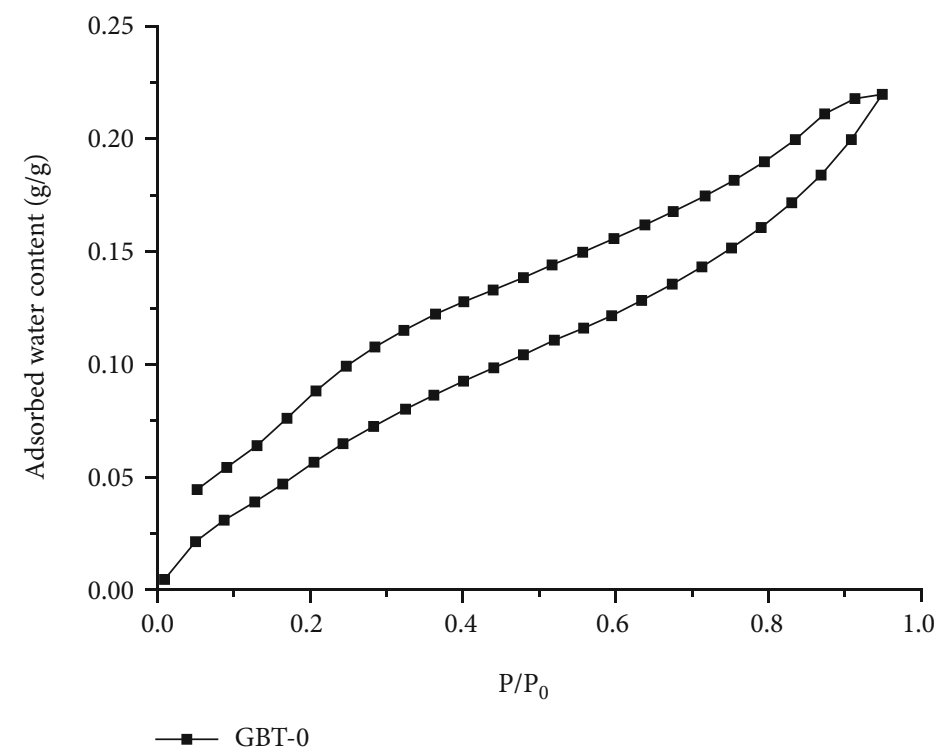

FIGURE 2: Water vapour adsorption isotherms of raw bentonite.

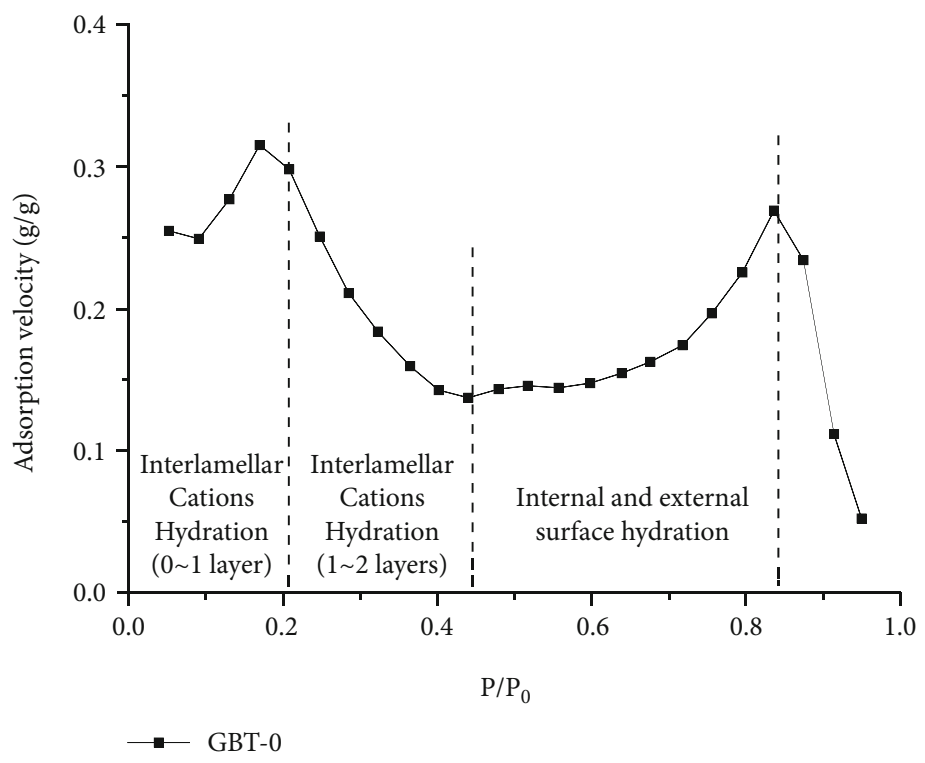

FIGURE 3: Water vapour adsorption velocity curves of raw bentonite.

mainly adsorbed on the basal surface of the crystal layer; and (4) when $\mathrm{RH}>0.8 \sim 0.9$, multilayer adsorption and even condensation occurred so that DDL water appeared.

\subsection{Definition of Multiscale Pores of Bentonite by Hydration} Status. The variation in pore characteristics in the hydration process was studied by the means mentioned in Section 2.7 and displayed in Figure 5. Figure 5(a) shows the curve of the void ratio versus $\mathrm{RH}$. Figure 5(b) illustrates the first derivative of the void ratio versus $\mathrm{RH}$, which represents the variational rate of pores in different hydration statuses. In Figure 5(a), bentonite with three kinds of dry densities gave a difference of void ratio in that a $1.4 \mathrm{~g} / \mathrm{cm}^{3}$ sample showed more notable increase of the void ratio with $\mathrm{RH}$ increasing at the range of $0 \sim 0.55$ than the others. An RH of $0 \sim 0.55$ was thought to be the range in which interlayer cations hydrated and crystalline swelling occurred. Bentonite with a $1.4 \mathrm{~g} / \mathrm{cm}^{3}$ dry density had fewer pores to accommodate the expansion of the crystal layer and finally resulted in an increase in macroscopic volume. Conversely, the samples with lower dry density had space to accept crystalline swelling so that the void ratio increased gently with $\mathrm{RH}$. Nevertheless, the results of bentonite with 3 dry densities all showed two boundaries, $\mathrm{RH} \approx 0.55$ and $\mathrm{RH} \approx 0.85$, at which the pore changed distinctly. Coincidentally, these two boundaries were close to the boundaries in the hydration mechanism. Specifically, in the range of $0<\mathrm{RH}<0.45 \sim$ 0.55 , water molecules were adsorbed around interlamellar cations, resulting in crystalline swelling. The interlayer spacing increased by approximately $5.4 \AA$, while the 


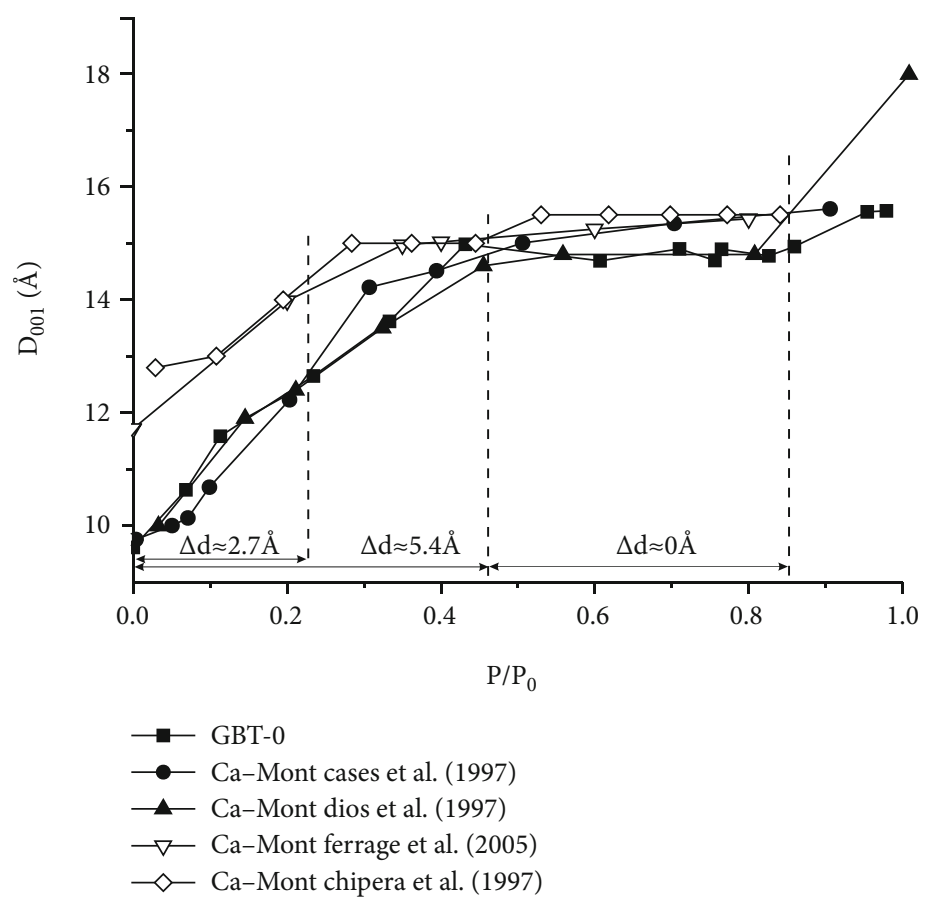

Figure 4: $d_{001}$ reflections versus $P / P_{0}$ for GBT and Ca-Monts.

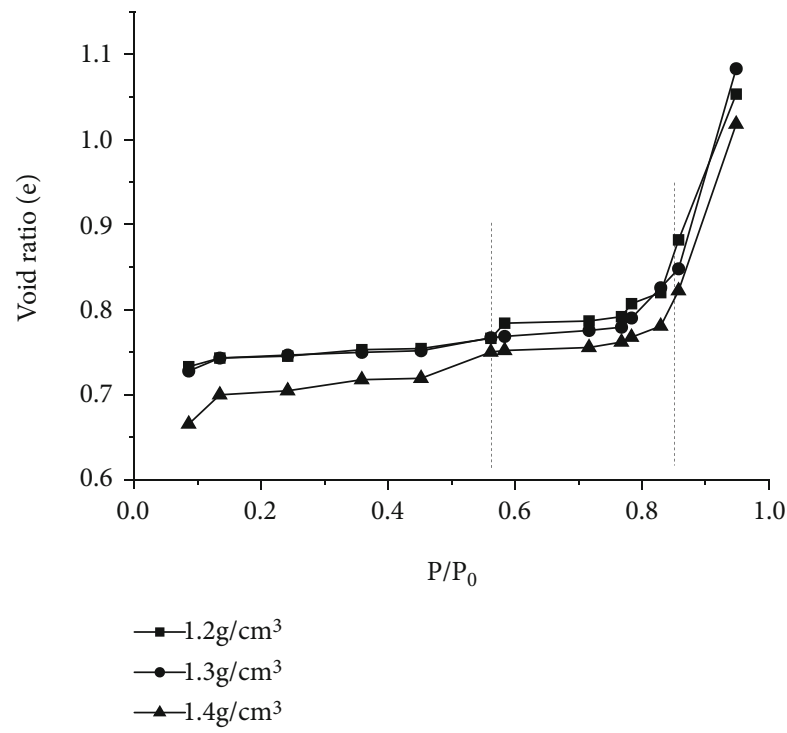

(a)

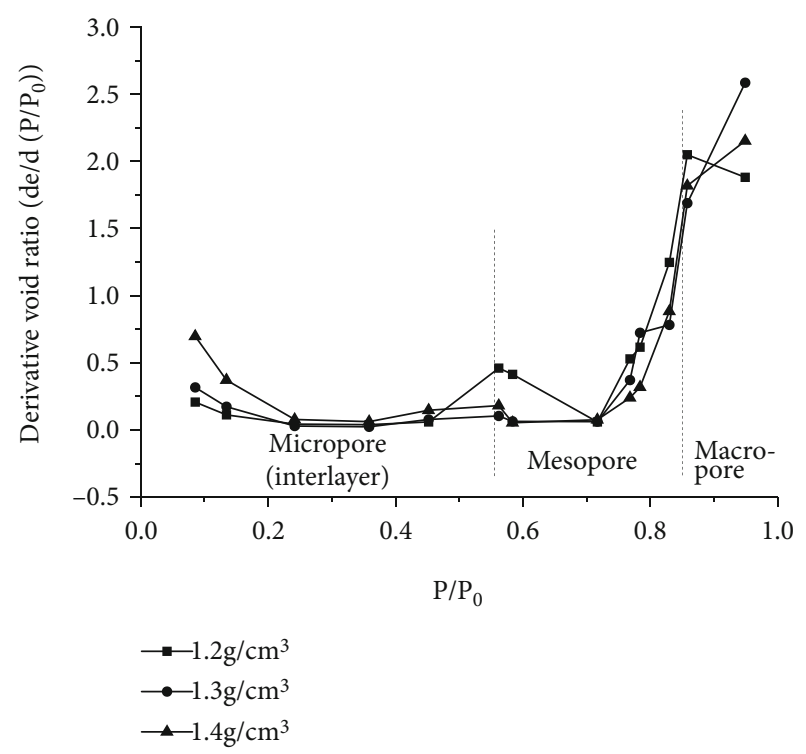

(b)

Figure 5: Void ratio in different $P / P_{0}$ for compacted GBT-0. (a) shows the curve with void ratio versus RH. (b) shows the first derivative of the void ratio versus $\mathrm{RH}$, which represents the variational rate of pores in different hydration statuses.

macrovolume showed indistinct changes. Based on the nomenclature terms of IUPAC, the adsorption of water in this range was considered to be conducted mainly by micropores in interlayers. In the range of $0.45 \sim 0.55<\mathrm{RH}<0.8$ $\sim 0.9$, water molecules entered the interlayer sequentially or were adsorbed on the external basal surface. For calcium bentonite, the external basal surface was formed by several single lamellae, and the combination was called a particle. In this hydration process, water molecules adsorbed on the basal surface started to fill in the pores among particles. This kind of pore was thought to be a mesopore $[5,6]$ so that $\mathrm{RH}$ $0.45 \sim 0.55$ was the boundary at which the mesopore began to adsorb water. Additionally, a peak for pore changing at $\mathrm{RH}$ $\approx 0.55$ is displayed in Figure 5(b), demonstrating that transformations of pore structures and water molecules started to enter mesopores. With further hydration at $\mathrm{RH}>0.8 \sim 0.9$, water molecules were continuously adsorbed on the external and internal surfaces, and some hydrated cations entered the 


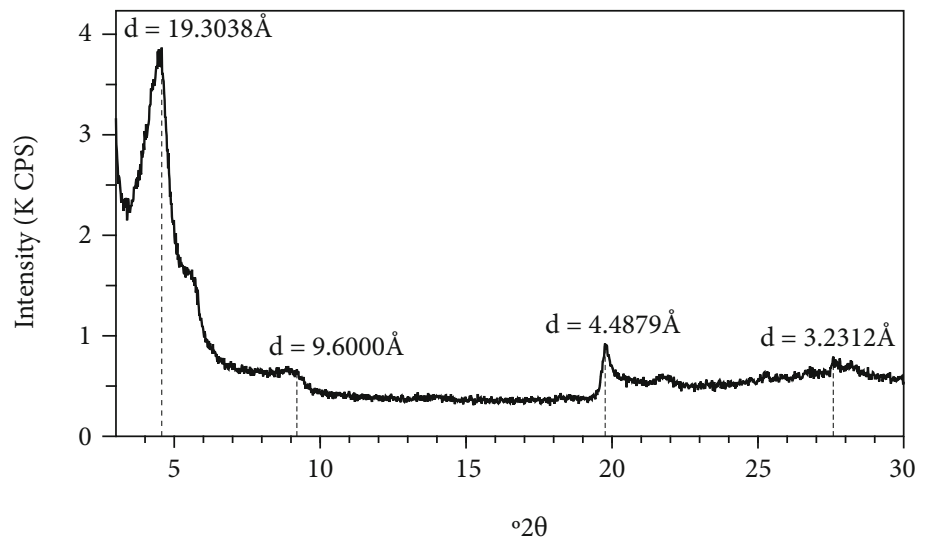

FIGURE 6: $d_{001}$ sampling of raw bentonite with water content up to the liquid limit.

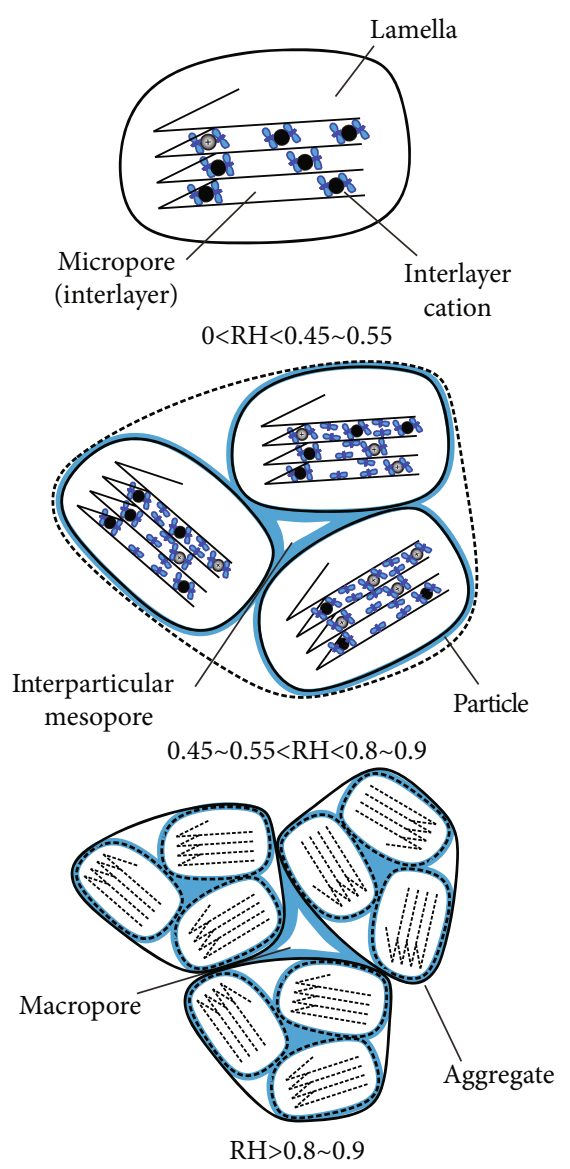

Figure 7: Hydration sequence in multiscale pores of calcium bentonite.

liquid phase to generate DDL around clay particles. Because of the concentration difference between micro- or mesopore fluids and soaking water from the outside, osmotic pressure appeared, driving water molecules to wedge into the pores among aggregates. In this hydration process, the distances among clay particles were strutted, and the sample exhibited a volume expansion so that the pore volume increased significantly when $\mathrm{RH}$ exceeded the boundary at approximately 0.85 . At the same time, the hydration energy of adsorbed water decreased rapidly (Figure 3 ) when $\mathrm{RH}>0.8 \sim 0.9$ (Figure 3) because water molecules needed to work to conquer the attractive force among clay particles and dissipate the hydration energy. Hence, when the $\mathrm{RH}$ was greater than $0.8 \sim 0.9$, aggregates increased in distance, and the pore volume expanded. Simultaneously, water molecules were adsorbed in these macropores $[5,6]$.

In particular, the statement about a certain pore starting to adsorb water meant water molecules mainly entered this kind of pore, which did not mean that water molecules could no longer enter the previous type of pore. For example, in the range of $0.45 \sim 0.55<\mathrm{RH}<0.8 \sim 0.9$, water molecules unceasingly entered the interlayer space (micropores) to be adsorbed on the basal surface. Additionally, in the process in which macropores adsorb water, the $d_{001}$ spacing expands from $15 \AA$ to $19.3 \AA$ when the water content reaches the liquid limit, as shown in Figure 6, indicating that micropores still adsorb water in this process.

In conclusion, the multiscale pores of calcium bentonite that adsorb water in different hydration processes were defined as follows: (1) in the range of $0<\mathrm{RH}<0.45 \sim 0.55$, interlamellar cations hydrated, and the micropores started to adsorb water, resulting in crystalline swelling; (2) in the range of $0.45 \sim 0.55<\mathrm{RH}<0.8 \sim 0.9$, water molecules got onto the basal surface of the crystal layer, and the mesopores began to adsorb water; and (3) in the range of $\mathrm{RH}>0.8 \sim 0.9$ , water molecules got onto the external and internal surfaces continuously to transform into DDL water, and the macropores initiated the adsorption of water. From this, a generalization diagram was finished in Figure 7 to display the hydration sequence in multiscale pores of calcium bentonite.

3.3. Influence of ISS on the Pore Characteristics of Bentonite. Figure 8 illustrates the water vapour adsorption isotherms of GBT-0, GBT-1:100, and GBT-1:50. The forms of the curves of the modified samples were similar to the forms of the curves of the raw sample. Meanwhile, the water vapour adsorption velocity curves shown in Figure 9 also displayed the same peaks, which meant that the modified bentonites had the same hydration mechanism and $\mathrm{RH}$ boundaries to define pore types as natural bentonite. Note that the adsorbance of raw samples was higher than the absorbance of 


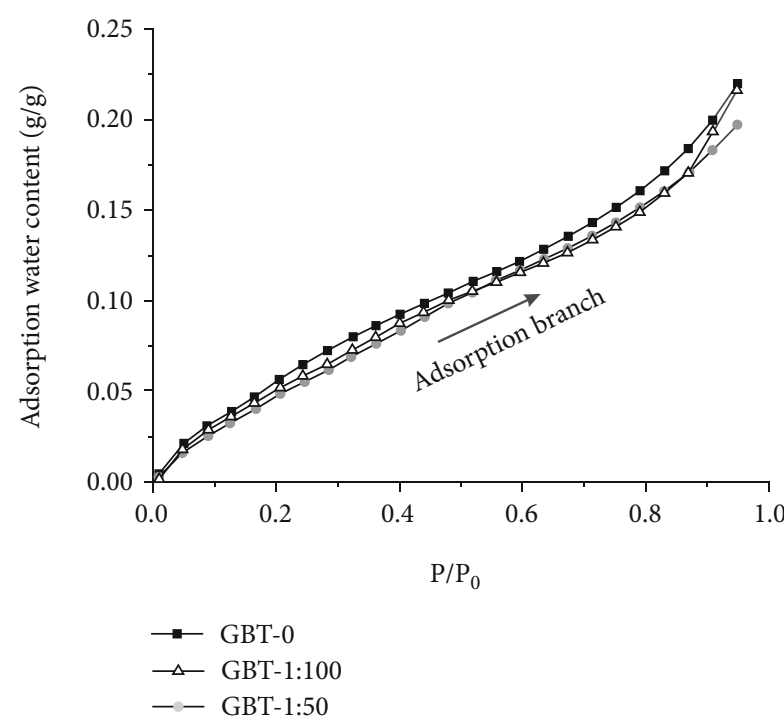

(a)

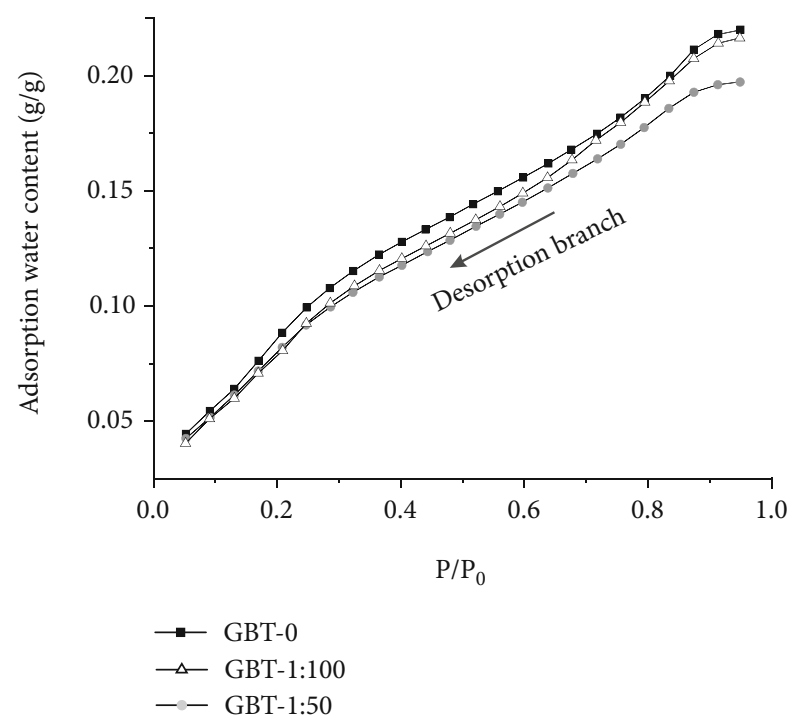

(b)

FIGURE 8: Water vapour adsorption isotherms of raw and modified bentonites.

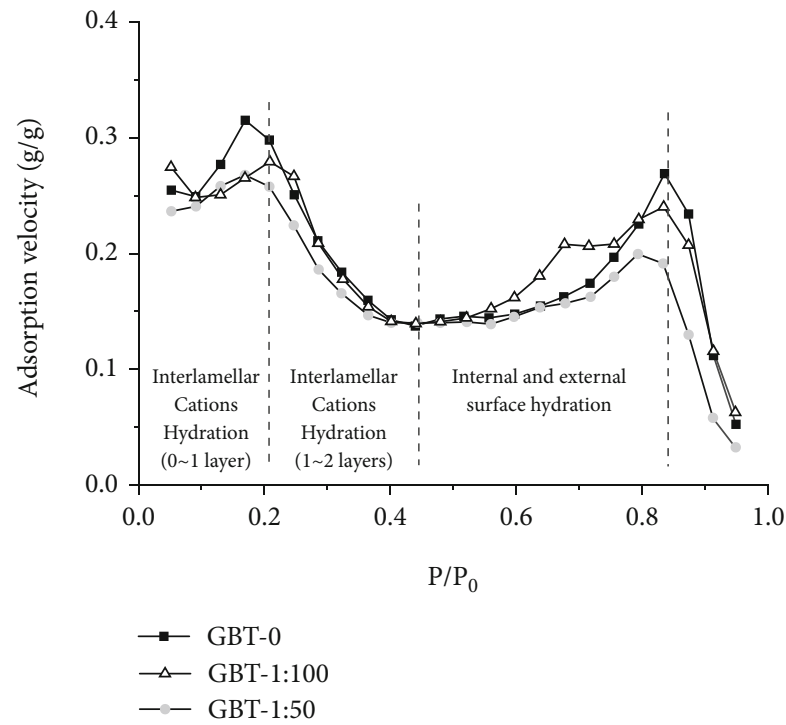

FIGURE 9: Water vapour adsorption velocity curves of raw and modified bentonites.

the modified samples, indicating that ISS reduced the adsorbing capacity of bentonite. Moreover, the isotherm of GBT-1: 100 displayed an increase in absorbed water content, which was close to the increase of GBT- 0 with an RH of $>0.9$ in the adsorption branch and $>0.7$ in the desorption branch, stating that the dilution ratios of ISS affected the reduction in adsorbed water content. In the water vapour adsorption test, sample GBT-1:100 showed a lower adsorbing capacity than the raw sample with a lower $\mathrm{RH}$, which meant that the ISS in bentonite was sufficient at the current time. However, with $\mathrm{RH}$ over 0.9 in the adsorption branch and 0.7 in the desorption branch, the ISS in GBT-1:100 might be insufficient, resulting in an approximation of the adsorbed water content. The interaction between ISS and clay min- erals was quite complex, which led to a difference in the water adsorbing capacity of modified bentonite.

3.3.1. Micropore Characteristics. Bentonite is a kind of clay that comprises phyllosilicate minerals. The specific structures of bentonite produced a phenomenon in which micropores were distributed mainly in the interlamellar space. As mentioned above, the range $0<\mathrm{RH}<0.45 \sim 0.55$ corresponded to the stage in which micropores adsorbed water. Therefore, adsorption equilibrium samples in an environment with $\mathrm{RH}=0.43$ were selected to measure the micropores (or interlayers) by XRD. Note that the measured micropore size was the result of adsorption equilibrium for $\mathrm{RH}=0.43$, which could not be used as the average value of micropores because the expansion of bentonite occurred during the range $0<\mathrm{RH}<0.45 \sim 0.55$. The results of raw and modified bentonites are shown in Figure 10(a), displaying a decrease in $d_{001}$ spacing from $14.9817 \AA$ to $14.7728 \AA$ for GBT-1: 100 and $14.6948 \AA$ for GBT-1:50 when ISS was added and demonstrating that the micropore size (or interlayer) of modified bentonite was reduced in comparison to the raw sample. At the stage in which micropores adsorbed water, the reduction in pore size resulted in a decrease in the adsorbed water content, as shown in Figure 2. Here, hydration and pore characteristics were demonstrated to be interdependent and reciprocal.

To deeply verify the above statement that the swelling behaviour of micropores was modified by ISS, adsorption equilibrium samples in saturated steam ethylene glycol (EG) were selected to test the $d_{001}$ spacing, as shown in Figure 10(b). EG was assumed to retain only one monolayer of EG molecules on the bentonite surface when samples were displayed in saturated steam [38], indicating the limit expansion of clay minerals and making the micropore size constant and measurable. Hence, the micropore size was used to investigate the characteristics of micropores. The result similarly showed that $d_{001}$ spacing of bentonite 


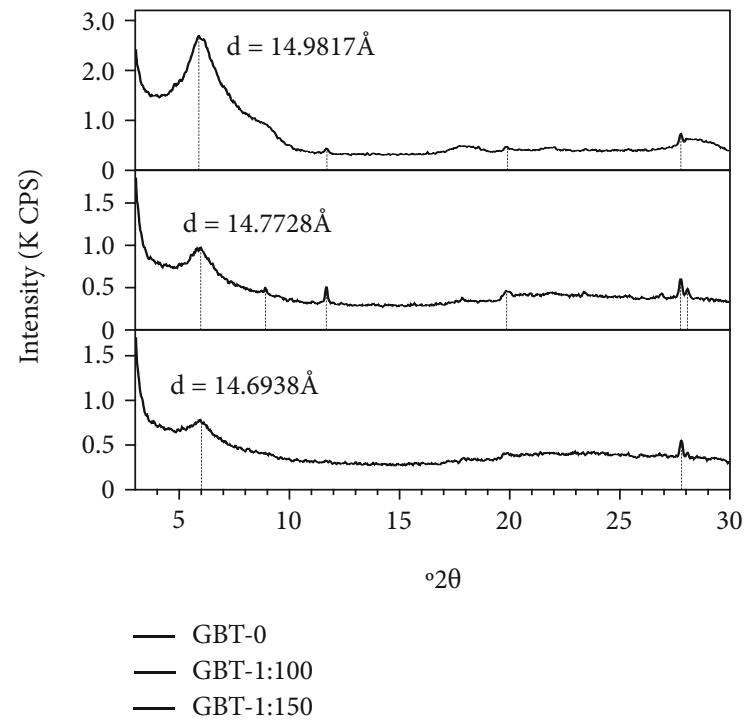

(a)

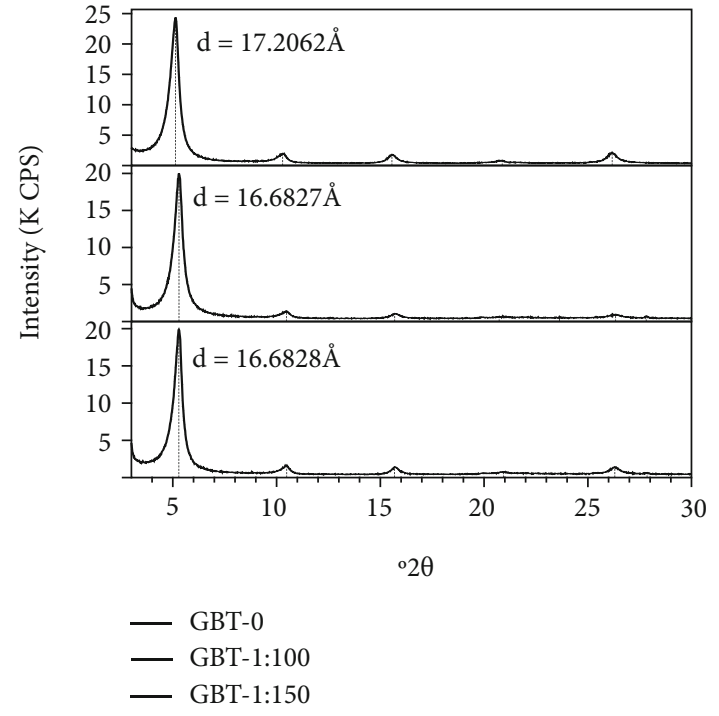

(b)

FIGURE 10: $d_{001}$ sampling of raw and modified bentonites that are equilibrated in (a) $R H=0.43$ and (b) saturated EG steam.

obviously decreased when it was modified by ISS and the values were from $17.2062 \AA$ to $16.6828 \AA$ and $16.6827 \AA$. However, the reduction of micropore size of the modified samples was not directly related to the ISS concentration, but instead showed similar values in Figure 10(b), which were analysed as follows: (a) the addition of the ISS caused the modified samples to become less homogeneous than the raw sample which made the measured micropore of modified bentonite with the same ISS concentration vary in size; (b) the reaction between the clay mineral and the ISS molecule was uneven and complicated, leading to difficulty in explaining the relationship between the ISS concentration and the $d_{001}$ spacing. Even so, the results in Figure 10(b) were sufficient to support the conclusion that ISS could reduce the micropore size, and in the hydration range of $0<\mathrm{RH}<0.45 \sim 0.55$, ISS had an inhibiting effect on the swelling behaviour of the micropores of bentonite.

3.3.2. Mesopore Characteristic. The mesopores were discussed through vapour adsorption based on certain pore analysis models. Specifically, the desorption branch was usually picked to analyse mesopore characteristics because it presented a clear hydration sequence, as mentioned above. With further hydration in the range of $0.45 \sim 0.55<\mathrm{RH}<$ $0.8 \sim 0.9$, the mesopores started to adsorb water. In this hydration process, water molecules were adsorbed on the external and internal surfaces of bentonite through van der Waals forces, making it possible to analyse the mesopore characteristics based on the D-H model presented by Dollimore and Heal [15]. The results based on water vapour adsorption are displayed in Figure 11, and the pore radii corresponding to boundary RHs of $0.45 \sim 0.55$ and $0.8 \sim 0.9$ were marked. In Figure 11, a peak was exhibited in the marked range presenting the main pore radii of bentonite samples. Therefore, the main mesopore radii of the modified samples were $6.0 \mathrm{~nm}$ (GBT-1:50) and $7.5 \mathrm{~nm}$ (GBT-1:100), in com- parison to $7.6 \mathrm{~nm}$ of the raw sample. Simultaneously, the corresponding volume of the main pore size decreased from $0.013 \mathrm{~cm}^{3} / \mathrm{g}$ to $0.011 \mathrm{~cm}^{3} / \mathrm{g}$ and $0.010 \mathrm{~cm}^{3} / \mathrm{g}$, demonstrating that clay particles were more aggregated.

In the discussion above, the analysis of mesopores was derived from the water vapour adsorption isotherm because this isotherm could present the mesopore characteristics in certain hydration sequences. However, measurement of mesopores is commonly performed by nitrogen adsorption considering that nitrogen molecules are nonpolar and interact with adsorbing materials through physical absorption. Allowing for a sufficient expansion of multiscale pores in the water content of the liquid limit for bentonite, pore structures in this status were conserved by means of freezedrying through liquid nitrogen, and the obtained bulk samples were applied to nitrogen adsorption tests to investigate mesopore characteristics. Figure 12(a) illustrates the nitrogen adsorption isotherms of bentonite samples, and Figure 12(b) shows the pore size distribution curves based on the D-H model. The nitrogen adsorption results demonstrated that the main pore radius of bentonite was in the range of $10 \mathrm{~nm} \sim 30 \mathrm{~nm}$ and that the pore radius of the modified samples decreased from $24.5 \mathrm{~nm}$ to $12.0 \mathrm{~nm}$ and $21.3 \mathrm{~nm}$ for GBT-1:100 and GBT-1:50, respectively. The volume that corresponded to the main pore radius of modified bentonites also displayed a reduction from $0.026 \mathrm{~cm}^{3} / \mathrm{g}$ to $0.025 \mathrm{~cm}^{3} / \mathrm{g}$ and $0.021 \mathrm{~cm}^{3} / \mathrm{g}$, indicating that mesopores changed and clay particles became more aggregate.

Note that an obvious and narrow peak was distributed around the $2 \mathrm{~nm}$ pore radius in Figure 12(b). Regarding this peak, it was said that it was a fake peak influenced by the diversity of pore types and connectivity of pore networks. Additionally, all the narrow peaks were distributed in the same pore radius $[39,40]$. Hence, this kind of peak is not discussed in this paper. 


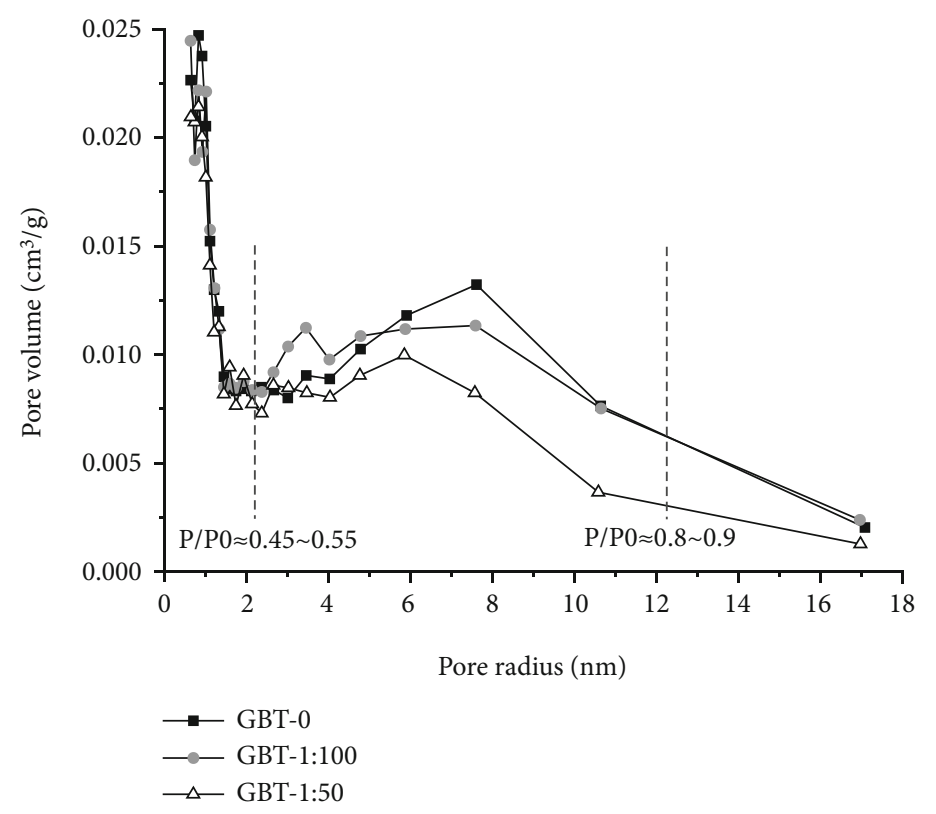

FIGURe 11: Pore size distribution of bentonites (water vapour adsorption).

3.3.3. Macropore Characteristics. As mentioned above, when $\mathrm{RH}>0.8 \sim 0.9$, the macropores started to adsorb water, and osmotic swelling occurred. At the same time, the hydration energy decreased, and the water transformed to DDL water. With further hydration and even adsorbed water from the liquid phase, the interparticle space swelled sustainably and obtained sufficient diffusion when the water content approached the liquid limit [41]. Simultaneously, the multiscale pores developed richly, and the average pore size could be presented by the thickness of the DDL water film. The thickness was calculated through the following equation [41]:

$$
h=\frac{W_{\mathrm{ext}}}{\operatorname{SSA}_{\mathrm{ext}} \times \rho_{\mathrm{w}}},
$$

where $\mathrm{SSA}_{\text {ext }}$ is the external specific surface area, $\rho_{\mathrm{w}}$ is the density of adsorbed water $\left(1 \mathrm{~g} / \mathrm{cm}^{3}\right)$, and $W_{\text {ext }}$ is the adsorbed water content of DDL water, which is calculated using the following equation:

$$
W_{\text {ext }}=W_{\mathrm{L}}-W_{0.9}
$$

where $W_{\mathrm{L}}$ is the water content of the liquid limit of bentonite and $W_{0.9}$ is the water content at $\mathrm{RH}=0.9$ adsorption equilibrium.

Figure 13 shows the liquid limit and specific surface area (SSA), including $\mathrm{SSA}_{\text {ext }}$, $\mathrm{SSA}_{\text {total }}$, and calculated $\mathrm{SSA}_{\text {int }}$. Based on the parameters, the thickness of the DDL water film is displayed in Table 3 through Equation (1). The results indicated that the thickness of the DDL of modified bentonite was reduced through the action of ISS and demonstrated that osmotic swelling was weakened, and the thickness of the adsorbed water film was thinned.
As a result of the liquid limit, GBT-1:100 presented a thinner DDL than GBT-1:50, implying that the wateradsorbed content of modified bentonite was not necessarily positively correlated with the applied ISS concentration. The same phenomenon has been found in earlier studies, and thus, the concept of the optimal ratio has been proposed $[27,28]$, which meant that more ISS did not necessarily produce a better modification result. Determining this optimal ratio requires a reference value for the physical and chemical properties, such as the liquid limit, free expansion rate, and CEC. The difference in the selection of the reference value would also cause the difference in the optimal ratio, and it was difficult to have a uniform reference value, so that all physical and chemical properties of the clay showed the same modification pattern as the ISS concentration. As a consequence, it could not be simply assumed that the higher the applied ISS concentration was, the greater the reduction in DDL thickness.

To measure the size of the macropores, a mercury injection test was implemented, as shown in Figure 14. Freezedrying was applied with the water content of the liquid limit before the test so that the pore structures could remain and be measured. The pore size distribution curves presented double peaks, indicating that two main macropores existed in bentonite. The main macropore size of the modified samples shifted from $9062 \mathrm{~nm}$ to $7248 \mathrm{~nm}$ (GBT-1:100) and $6583 \mathrm{~nm}$ (GBT-1:50) and from $2543 \mathrm{~nm}$ to $1624 \mathrm{~nm}$ (GBT-1:100) and $2509 \mathrm{~nm}$ (GBT-1:50) in comparison to the raw sample. Having the same changing trend as microand mesopores, modified samples swelled limitedly when hydration and development of macropores were depressed under the influence of ISS.

3.4. The Origin of Adsorbed Water and Pore Structures Changing by Adding ISS. RHs of $0.45 \sim 0.55$ and $0.8 \sim 0.9$ were defined as the boundaries of pore types. For modified 


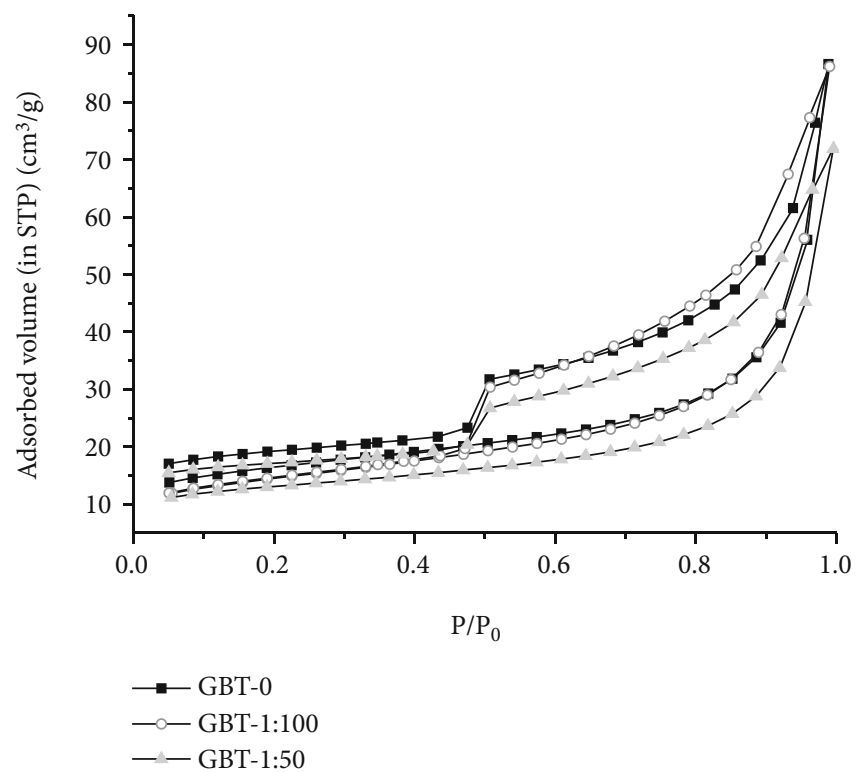

(a)

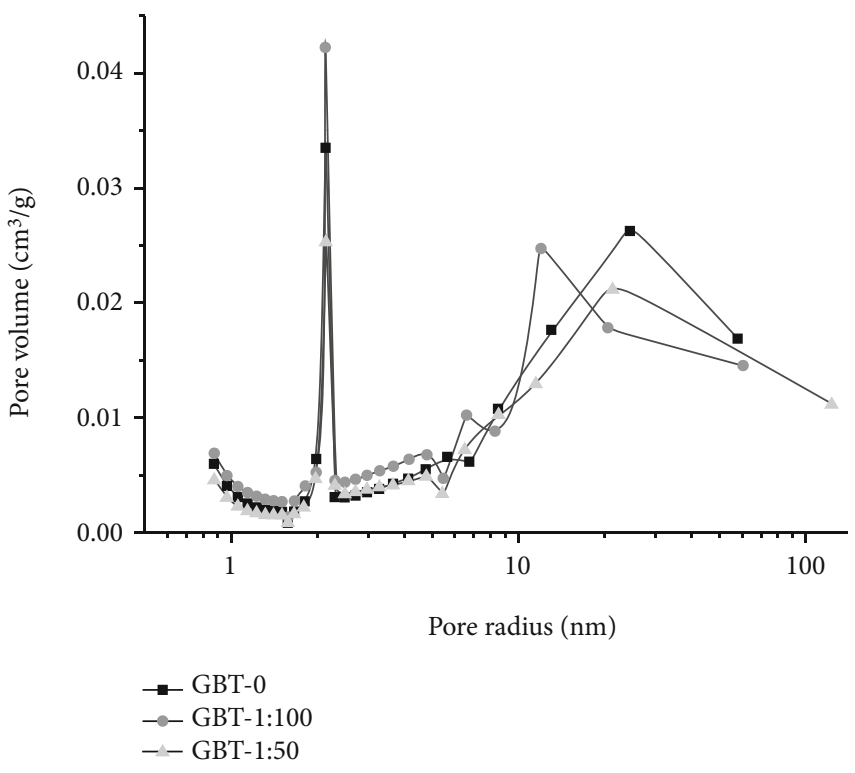

(b)

Figure 12: Pore size distribution of bentonites (nitrogen adsorption).

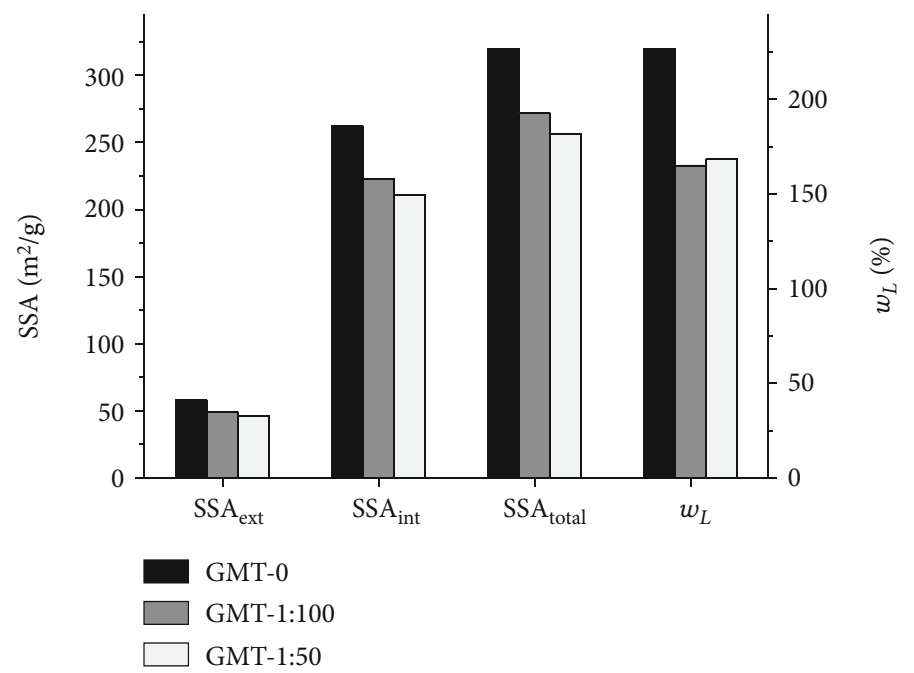

FIGURE 13: SSA and liquid limit of bentonites.

TABLE 3: Thickness of weak adsorption water for bentonites.

\begin{tabular}{lccc}
\hline Samples & GBT-0 & GBT-1 $: 100$ & GBT-1:50 \\
\hline$h(\mathrm{~nm})$ & 35.78 & 29.61 & 32.68 \\
\hline
\end{tabular}

samples, the curve forms were similar to the curve forms of the raw sample, also having the same peaks shown in Figure 9. Therefore, boundaries of $0.45 \sim 0.55$ and $0.8 \sim 0.9$ were used to define the pore types of modified samples, such as natural bentonite. In the process in which micropores adsorbed water $(0<\mathrm{RH}<0.45 \sim 0.55)$, the water content of modified bentonite decreased in comparison to raw bentonite, as shown in Figure 8. In this hydration process, the micropore size also decreased, as shown in Figure 10, show- ing that changing the adsorbed water influenced the expansion of micropores. For the reason why adsorbed water decreased in this range, the hydration mechanism pointed out that it resulted from the decrease of the cation amount, shown in Figure 15, suggesting ISS regulated adsorbed water in micropores through exchangeable cations and finally influenced the size of the micropore.

With further hydration in the range $0.45 \sim 0.55<\mathrm{RH}<$ $0.8 \sim 0.9$, the content of adsorbed water in mesopores was reduced for modified bentonites, as illustrated in Figure 8 . Meanwhile, the mesopores shrank, as shown in Figures 11 and 12. In addition to the influence that adsorbed water had on micropores, mesopores were also affected by the content of adsorbed water. In this range, the basal surface of the crystalline layer adsorbed water molecules, obviously 


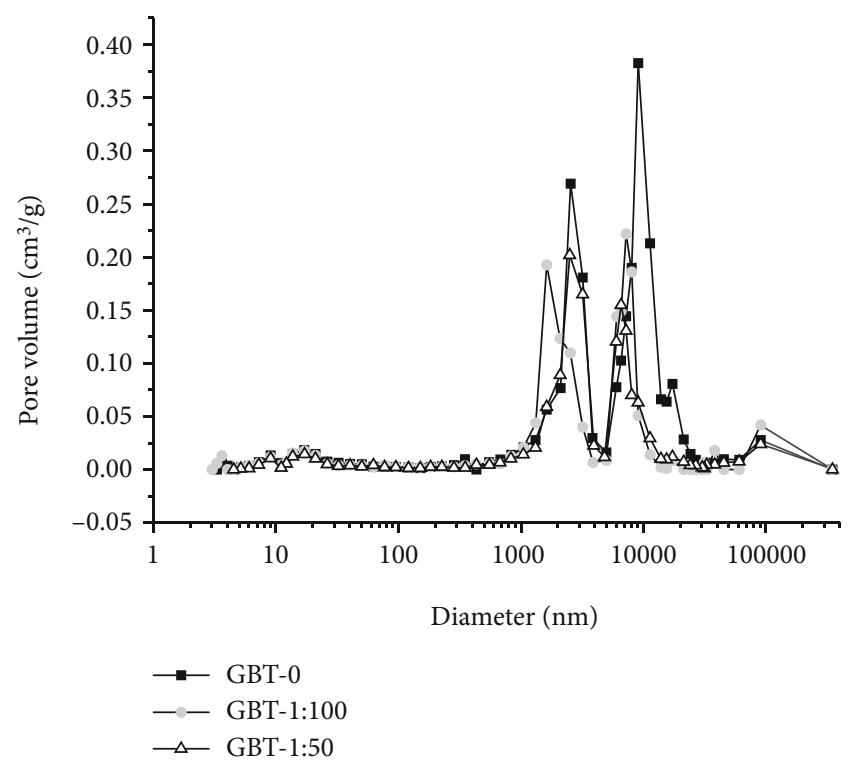

Figure 14: Pore size distribution of bentonites (by mercury intrusion).

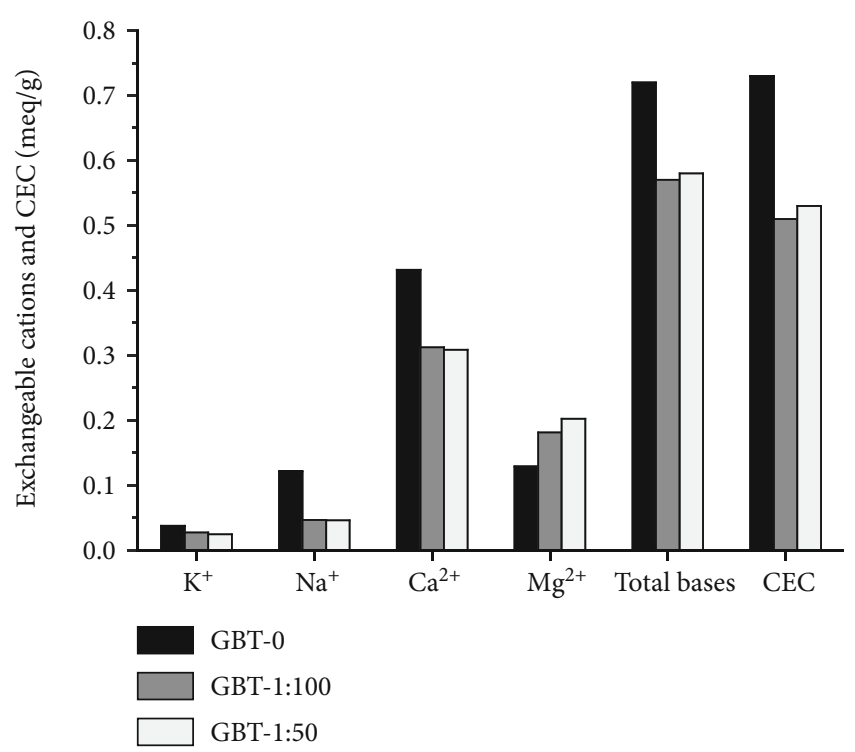

FIgURE 15: Exchangeable cations and CEC of samples.

showing that the SSA was an important index to evaluate the hydratability of bentonite. Figure 13 illustrates the SSA of modified and raw samples, presenting a decrease in SSA for modified bentonites in comparison to natural bentonites, which pointed out the reason why water in mesopores decreased. Therefore, the swelling of mesopores in the modified sample was limited.

When $\mathrm{RH}$ was higher than $0.8 \sim 0.9$ and even adsorbing water was in the liquid phase, water entered macropores, and the water content decreased for modified bentonites, as shown in Figure 8. Simultaneously, the size of the macropores decreased, as shown in Figure 14 and Table 3, demonstrating that macropores had a direct connection with adsorbed water. In this hydration process, the decrease in adsorbed water was produced by the comprehensive influence of SSA and CEC reduction. The expansion of macropores in modified bentonite was not sufficient because of the limit of adsorbed water.

From the above, regulation of ISS was brought to adsorbed water, and pore structures were realized through the reduction of exchangeable cations and the basal surface. In certain hydration sequences, ISS regulated the corresponding dominant hydration factors.

\section{Conclusions}

The hydration process and pore structure expansion were reciprocal. ISS regulated the corresponding dominant hydration factors to modify the adsorbed water and pore characteristics in certain hydration sequences. In this paper, water vapour and nitrogen adsorption, the mercury intrusion method, XRD, the exchangeable cation test, and the Atterberg limit test were implemented to investigate the hydration mechanism and pore structure evolution of bentonite. The $\mathrm{RH}$ was raised to define the hydration sequence and multiscale pore structures. In the range of $0<\mathrm{RH}<$ $0.45 \sim 0.55$, interlamellar cations hydrated and crystalline swelling occurred. In this range, water molecules entered the interlayer space, resulting in the expansion of micropores. Moreover, when $0.45 \sim 0.55<\mathrm{RH}<0.8 \sim 0.9$, water molecules were adsorbed on the basal surface of the crystal layer. Correspondingly, the mesopores started to adsorb water. With further hydration when $\mathrm{RH}>0.8 \sim 0.9$, the surface of clay particles adsorbed water molecules sequentially, and water transformed into DDL water. Simultaneously, osmotic swelling occurred, and macropores expanded because of water adsorption. By adding ISS to bentonite, the sizes of micro-, meso-, and macropores all decreased because adsorbed water in multiscale pores was modified. ISS regulated adsorbed water to influence pore structure by modifying microparameters. In the hydration process in which interlamellar cations hydrated, ISS weakened the interaction between cations and water molecules to influence the micropore structure. In the process by which the basal surface of the crystal layer adsorbs water, ISS reduces the surface hydration energy to influence the mesopore structure. In the process in which osmotic swelling occurred, the DDL water was influenced by the reduction of cations and the basal surface, and the macropores changed concomitantly.

However, the quantitative relationship between ISS concentration and the reduction of water retention and pore size has not been investigated. To understand the precise application of ISS, further study will be necessary to establish such a functional relationship.

\section{Data Availability}

A data availability statement has been included in my manuscript. 


\section{Conflicts of Interest}

The authors declare that they have no conflicts of interest.

\section{Acknowledgments}

This work was supported by the Education Reform Project of the Ministry of Education (E2020040), Sichuan Science and Technology Program (No. 2019JDRC0109), and National Natural Science Foundation of China (No. 41672297).

\section{References}

[1] D. A. Laird, "Influence of layer charge on swelling of smectites," Applied Clay Science, vol. 34, no. 1-4, pp. 74-87, 2006.

[2] L. Liu, "Prediction of swelling pressures of different types of bentonite in dilute solutions," Colloids and Surfaces A: Physicochemical and Engineering Aspects, vol. 434, no. 19, pp. 303318, 2013.

[3] F. T. Madsen and M. Müller-Vonmoos, "The swelling behaviour of clays," Applied Clay Science, vol. 4, no. 2, pp. 143156, 1989.

[4] K. Norrish, "The swelling of montmorillonite," Discussions of the Faraday Society, vol. 18, pp. 120-134, 1954.

[5] F. Salles, I. Beurroies, O. Bildstein et al., "A calorimetric study of mesoscopic swelling and hydration sequence in solid Namontmorillonite," Applied Clay Science, vol. 39, no. 3-4, pp. 186-201, 2008.

[6] F. Salles, O. Bildstein, J. Douillard, M. Jullien, J. Raynal, and H. van Damme, "On the cation dependence of interlamellar and interparticular water and swelling in smectite clays," Langmuir, vol. 26, no. 7, pp. 5028-5037, 2010.

[7] D. Feng, X. Li, X. Wang et al., "Water adsorption and its impact on the pore structure characteristics of shale clay," Applied Clay Science, vol. 155, pp. 126-138, 2018.

[8] D. A. Laird, "Model for crystalline swelling of 2:1 phyllosilicates," Clays and Clay Minerals, vol. 44, no. 4, pp. 553-559, 1996.

[9] M. Tuller and D. Or, "Hydraulic functions for swelling soils: pore scale considerations," Journal of Hydrology, vol. 272, no. 1-4, pp. 50-71, 2003.

[10] F. Salles, J. Douillard, R. Denoyel et al., "Hydration sequence of swelling clays: evolutions of specific surface area and hydration energy," Journal of Colloid and Interface Science, vol. 333, no. 2, pp. 510-522, 2009.

[11] I. Berend, J. M. Cases, M. Francois et al., "Mechanism of adsorption and desorption of water vapor by homoionic montmorillonites; 2 , the $\mathrm{Li}^{+}, \mathrm{Na}^{+}, \mathrm{K}^{+}, \mathrm{Rb}^{+}$and $\mathrm{Cs}^{+}$-exchanged forms," Clays and Clay Minerals, vol. 43, no. 3, pp. 324-336, 1995.

[12] J. M. Cases, I. Berend, G. Besson et al., "Mechanism of adsorption and desorption of water vapor by homoionic montmorillonite. 1. The sodium-exchanged form," Langmuir, vol. 8, no. 11, pp. 2730-2739, 1992.

[13] J. M. Cases, I. Berend, M. Francois, L. P. Uriot, L. J. Michot, and F. Thomas, "Mechanism of adsorption and desorption of water vapor by homoionic montmorillonite: 3. The $\mathrm{Mg}^{2+}$, $\mathrm{Ca}^{2+}, \mathrm{Sr}^{2+}$ and $\mathrm{Ba}^{2+}$ exchanged forms," Clays and Clay Minerals, vol. 45, no. 1, pp. 8-22, 1997.
[14] E. P. Barrett, L. G. Joyner, and P. P. Halenda, “The determination of pore volume and area distributions in porous substances I. Computations from Nitrogen Isotherms," Journal of the American Chemical Society, vol. 73, no. 1, pp. 373-380, 1951.

[15] D. Dollimore and G. R. Heal, "Pore-size distribution in typical adsorbent systems," Journal of Colloid and Interface Science, vol. 33, no. 4, pp. 508-519, 1970.

[16] E. W. Washburn, "Note on a method of determining the distribution of pore sizes in a porous material," Proceedings of the National Academy of Sciences of the United States of America, vol. 7, no. 4, pp. 115-116, 1921.

[17] R. W. Mooney, A. G. Keenan, and L. A. Wood, “Adsorption of water vapor by montmorillonite II. Effect of exchangeable ions and lattice swelling as measured by X-ray diffraction," Journal of the American Chemical Society, vol. 74, no. 6, pp. 13711374, 1952.

[18] M. al-Mukhtar, A. Lasledj, and J. Alcover, "Behaviour and mineralogy changes in lime-treated expansive soil at $50^{\circ} \mathrm{C}$," Applied Clay Science, vol. 50, no. 2, pp. 199-203, 2010.

[19] S. H. Chew, A. H. M. Kamruzzaman, and F. H. Lee, "Physicochemical and engineering behavior of cement treated clays," Journal of Geotechnical and Geoenvironmental Engineering, vol. 130, no. 7, pp. 696-706, 2004.

[20] S. M. Rao, B. V. V. Reddy, and M. Muttharam, "The impact of cyclic wetting and drying on the swelling behaviour of stabilized expansive soils," Engineering Geology, vol. 60, no. 1-4, pp. 223-233, 2001.

[21] F. Yazdandoust and S. S. Yasrobi, "Effect of cyclic wetting and drying on swelling behavior of polymer-stabilized expansive clays," Applied Clay Science, vol. 50, no. 4, pp. 461-468, 2010.

[22] D. E. Scholen, "Stabilizer mechanisms in nonstandard stabilizers," in Proceedings of Sixth International Conference on Low-Volume Roads, Minneapolis, 1995.

[23] L. E. Katz, A. F. Rauch, H. M. Liljestrand, J. S. Harmon, K. S. Shaw, and H. Albers, "Mechanisms of soil stabilization with liquid ionic stabilizer," Transportation Research Record, vol. 1757, no. 1, pp. 50-57, 2001.

[24] Q. B. Liu, W. Xiang, D. S. Cui, and L. J. Cao, "Mechanism of expansive soil improved by ionic soil stabilizer," Chinese Journal of Geotechnical Engineering, vol. 33, no. 4, pp. 648-654, 2011.

[25] T. Petry and B. Das, "Evaluation of chemical modifiers and stabilizers for chemically active soils-clays," Transportation Research Record, vol. 1757, no. 1, pp. 43-49, 2001.

[26] A. Rauch, J. Harmon, L. Katz, and H. Liljestrand, "Measured effects of liquid soil stabilizers on engineering properties of clay," Transportation Research Record, vol. 1787, no. 1, pp. 33-41, 2002.

[27] D. S. Cui, Research on the reaction mechanism of adsorbed water in red clay of Wuhan with ionic soil stabilizer, [Ph.D. thesis], China University of Geosciences, Wuhan, 2009.

[28] X. S. Lu and W. Xiang, "Experimental study on dynamic characteristics of ionic soil stabilizer reinforcing red clay," Advanced Materials Research, vol. 374-377, pp. 1391-1395, 2011.

[29] M. Khorshidi and N. Lu, "Intrinsic relation between soil water retention and cation exchange capacity," Journal of Geotechnical and Geoenvironmental Engineering, vol. 143, no. 4, article 04016119, 2017. 
[30] K. Devineau, I. Bihannic, L. Michot et al., "In situ neutron diffraction analysis of the influence of geometric confinement on crystalline swelling of montmorillonite," Applied Clay Science, vol. 31, no. 1-2, pp. 76-84, 2006.

[31] D. A. Laird and C. Shang, "Relationship between cation exchange selectivity and crystalline swelling in expanding 2:1 phyllosilicates," Clays and Clay Minerals, vol. 45, no. 5, pp. 681-689, 1997.

[32] F. Salles, O. Bildstein, J. Douillard, M. Jullien, and H. van Damme, "Determination of the driving force for the hydration of the swelling clays from computation of the hydration energy of the interlayer cations and the clay layer," The Journal of Physical Chemistry C, vol. 111, no. 35, pp. 13170-13176, 2007.

[33] A. Revil and N. Lu, "Unified water isotherms for clayey porous materials," Water Resources Research, vol. 49, no. 9, pp. 56855699, 2013.

[34] W. F. Woodruff and A. Revil, "CEC-normalized clay-water sorption isotherm," Water Resources Research, vol. 47, no. 11 , pp. 553-561, 2011.

[35] S. J. Chipera, J. W. Carey, and D. L. Bish, "Controlled-humidity XRD analyses: application to the study of smectite expansion/contraction," Advances in X-Ray Analysis, vol. 39, pp. 713-722, 1995.

[36] G. Dios Cancela, F. J. Huertas, E. Romero Taboada, F. Sánchez-Rasero, and A. Hernández Laguna, "Adsorption of water vapor by homoionic montmorillonites. Heats of adsorption and desorption," Journal of Colloid and Interface Science, vol. 185, no. 2, pp. 343-354, 1997.

[37] E. Ferrage, B. Lanson, B. A. Sakharov, and V. A. Drits, "Investigation of smectite hydration properties by modeling experimental X-ray diffraction patterns: part I. Montmorillonite hydration properties," American Mineralogist, vol. 90, no. 89, pp. 1358-1374, 2005.

[38] R. S. Dyal and S. B. Hendricks, "Total surface of clays in polar liquids as a characteristic index," Soil Science, vol. 69, no. 6, pp. 503-509, 1950.

[39] J. C. Groen, L. A. A. Peffer, and J. Pérez-Ramírez, "Pore size determination in modified micro- and mesoporous materials. Pitfalls and limitations in gas adsorption data analysis," Microporous and Mesoporous Materials, vol. 60, no. 1-3, pp. 1-17, 2003.

[40] J. C. Groen, J. Pérez-Ramírez, and L. A. A. Peffer, "Formation of uniform mesopores in ZSM-5 zeolite upon alkaline posttreatment?," Chemistry Letters, vol. 31, no. 1, pp. 94-95, 2002.

[41] B. Dolinar and B. Macuh, "Determining the thickness of adsorbed water layers on the external surfaces of clay minerals based on the engineering properties of soils," Applied Clay Science, vol. 123, no. 35, pp. 279-284, 2016. 